\title{
Influence of a Hairpin Loop on the Thermodynamic Stability of a DNA Oligomer
}

\author{
Jurij Lah, Mojca Seručnik, and Gorazd Vesnaver \\ Faculty of Chemistry and Chemical Technology, University of Ljubljana, Askerceva 5, 1000 Ljubljana, Slovenia \\ Correspondence should be addressed to Jurij Lah, jurij.lah@fkkt.uni-lj.si and Gorazd Vesnaver, gorazd.vesnaver@fkkt.uni-lj.si \\ Received 13 April 2011; Accepted 16 May 2011 \\ Academic Editor: Luis A. Marky \\ Copyright () 2011 Jurij Lah et al. This is an open access article distributed under the Creative Commons Attribution License, which \\ permits unrestricted use, distribution, and reproduction in any medium, provided the original work is properly cited. \\ DSC was used to evaluate the mechanism of the thermally induced unfolding of the single-stranded hairpin $\mathrm{HP}=5^{\prime}$ - \\ CGGAATTCCGTCTCCGGAATTCCG-3' and its core duplex D = (5'-CGGAATTCCG-3' $)_{2}$. The DSC melting experiments \\ performed at several salt concentrations were successfully described for HP and D in terms of a three-state transition model \\ $\mathrm{HP} \leftrightarrow \mathrm{I}$ (intermediate state) $\leftrightarrow \mathrm{S}$ (unfolded single-stranded state) and two state transition model D $\leftrightarrow 2 \mathrm{~S}$, respectively. Comparison \\ of the model-based thermodynamic parameters obtained for each HP and D transition shows that in unfolding of HP only the \\ $\mathrm{HP} \leftrightarrow \mathrm{I}$ transition is affected by the TCTC loop. This observation suggests that in the intermediate state its TCTC loop part exhibits \\ significantly more flexible structure than in the folded state while its duplex part remains pretty much unchanged.
}

\section{Introduction}

Hairpin loops are a common form of nucleic acid secondary structure and are crucial for tertiary structure and function [1]. They are known to play a key role in a number of biological processes such as gene expressions, DNA recombination, and DNA transposition [2-4]. In RNA molecules hairpins act as nucleation sites for RNA folding into final conformations [5-7] and play a critical role in RNA-protein recognition and gene regulation $[8,9]$. Furthermore, due to the specificity of probe/target hybridization determined as a match-versus-mismatch discrimination, hairpin DNA oligomer probes have become an important tool in modern biotechnology and diagnostics $[10,11]$. The thermodynamics and kinetics of hairpin formation, hairpin binding to complementary nucleic acids, and hairpin-ligand associations have been studied extensively [12-21]. There is no doubt that studies of hairpin-to-coil transitions and hairpinligand binding affinity and specificity have greatly enhanced our understanding of structural features and function of the naturally occurring nucleic acids [22, 23]. However, despite extensive biophysical research on the systems involving hairpin structures that produced a number of high-quality explanations and evaluations on properties and behavior of nucleic acids containing hairpin formations, there are still many unresolved questions.
As pointed out by Marky et al. [24] the most suitable hairpin molecules for studying the thermodynamics of their conformational transitions and ligand binding are the singlestranded hairpin molecules. They form stable partially paired duplexes that tend to melt in simple monomolecular transitions. Furthermore, their conformational stability and ligand binding properties are easily compared with those of the corresponding core duplexes. In this way one can evaluate the contributions of the loops to the thermal stability of the hairpins. Despite the simple structure of single-stranded hairpins it is not clear whether their monomolecular folding/unfolding transitions occur in a two-state or multistate manner. Measurements of their thermally induced unfolding transitions followed by UV, CD, and/or fluorescence spectroscopy as a rule result in sigmoidal melting curves suggesting that they may be considered as two-state processes. The same conclusion can be reached also on the basis of DSC measurements performed on the same sample solutions in the older generation of less sensitive DSC instruments (e.g., Microcal MC-2) which resulted in single-peak DSC thermograms. Recent measurements of conformational transitions of DNA quadruplex structures have shown, however, that the sigmoidal shape of UV or CD melting curves may be misleading. Namely, the DSC measurements performed on samples for which sigmoidal UV and CD melting curves were 


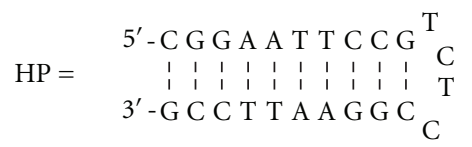

$$
\mathrm{D}=\begin{array}{r}
5^{\prime}-\mathrm{C} \\
\mathrm{L}
\end{array}
$$

FIGURE 1: Schematic presentation of the model oligonucleotides: hairpin (HP), duplex (D).

observed using the DSC of the latest generation (CSC, Microcal) resulted in thermograms containing two or three well-distinguished peaks thus indicating that the observed DNA melting process occurs in a multi-state manner [25, 26]. Furthermore, recent T-jump experiments performed on small hairpin molecules have produced a direct evidence that their unfolding transitions involve intermediate structures and thus cannot be considered as two-state processes [19, 2729].

In our DSC study of the unfolding mechanism and stability of the $5^{\prime}$-CGGAATTCCGTCTCCGGAATTCCG-3' hairpin we performed the DSC melting experiments on the hairpin and its core duplex, (5'-CGGAATTCCG-3') ${ }_{2}$ (see Figure 1), at several salt concentrations using an extremely sensitive microcalorimeter (CSC). To see to what extent the TCTC loop affects the hairpin unfolding process we attempted to describe for each oligonucleotide the measured DSC thermograms in terms of the simplest possible unfolding model. We derived the corresponding model functions and by fitting them to the experimental data we tested the appropriateness of the suggested models and obtained for each transition the characteristic thermodynamic quantities of transition $\Delta G_{(T)}^{0}, \Delta H_{(T)}^{0}, \Delta S_{(T)}^{0}$, and $\Delta c_{P}^{0}$. By comparing these values determined for the hairpin and the core duplex we tried to estimate the contribution of the TCTC loop to the stability of the hairpin.

\section{Materials and Methods}

2.1. Materials. Self-complementary oligonucleotide $5^{\prime}$-CGGAATTCCG- $3^{\prime}$ and oligonucleotide $5^{\prime}$-CGGAATTCCGTCTCCGGAATTCCG-3' $3^{\prime}$ that in solution at room temperature form a duplex (D) and a single-stranded hairpin structure (HP), respectively, were purchased HPLC pure from Invitrogen Co., Germany and used without any further purification. Their concentrations in buffer solution $(10 \mathrm{mM}$ phosphate buffer and $1 \mathrm{mM} \mathrm{Na} 2$ EDTA adjusted to $\mathrm{pH}=$ 7.0)) in the presence of $100 \mathrm{mM} \mathrm{NaCl}$ were determined at $25^{\circ} \mathrm{C}$ spectrophotometrically in the Cary Bio $100 \mathrm{UV}$-spectrophotometer. The molar extinction coefficients were determined using the nearest neighbor data of Cantor et al. [30] for single-stranded DNA at $25^{\circ} \mathrm{C}$ and the absorbance at $260 \mathrm{~nm}$ of thermally unfolded oligonucleotide extrapolated back to $25^{\circ} \mathrm{C}\left(\varepsilon_{\mathrm{D} 260}=84600 \mathrm{M}^{-1} \mathrm{~cm}^{-1}, \varepsilon_{\mathrm{HP} 260}=\right.$ $\left.216000 \mathrm{M}^{-1} \mathrm{~cm}^{-1}\right)$. The phosphate buffer solutions used in all experiment contained $0,0.1,0.3$, or $1.0 \mathrm{M} \mathrm{NaCl}$.

Differential scanning calorimetry (DSC). Thermally induced unfolding of duplex (D) and hairpin $(\mathrm{H})$ in buffer solutions with different added $\mathrm{NaCl}$ concentrations was followed between 5 and $95^{\circ} \mathrm{C}$ in a Nano-II DSC calorimeter (CSC; UT) at the heating rate of $1^{\circ} \mathrm{C} / \mathrm{min}$ and essentially the same results were obtained from several test-experiments performed at the heating rate of $0.25^{\circ} \mathrm{C} / \mathrm{min}$. The thermally induced unfolding of both oligonucleotides was monitored in terms of $c_{P_{\mathrm{ex}}}=\bar{c}_{p_{2}}-\bar{c}_{p_{D, F}}$ versus $T$ thermograms in which the differences between the partial molar heat capacity of the measured oligonucleotide $\bar{c}_{p_{2}}$ (raw signals corrected for the solvent contributions) and the partial molar heat capacities of the corresponding folded states extrapolated from low temperatures over the whole measured temperature interval, $\bar{c}_{p_{D, F}}$, are normalized for the duplex or hairpin concentration. The total enthalpy of unfolding, $\Delta H_{(T)}^{\text {cal }}$, was obtained from the measured thermograms as the area under the $c_{P_{e x}(T)}$ versus $T$ curve.

2.2. Analysis of the DSC Thermograms. The thermally induced conformational transitions can be experimentally followed in a model-independent way only by DSC. At relatively low concentrations used in DSC experiments the measured solute-normalized heat capacity of the sample solution, $c_{P(T)}$, with the subtracted baseline may be equalized with the oligonucleotide partial molar heat capacity, $\bar{c}_{P_{2}(T)}$. Thus, the overall heat effect that accompanies the measured conformational transition from its initial folded state at the temperature $T_{1}$ to its final unfolded state at $T_{2}$ can be expressed as

$$
\Delta H_{\left(T_{1} \rightarrow T_{2}\right)}=\int_{T_{1}}^{T_{2}} \bar{c}_{p_{2}(T)} d T .
$$

Since the enthalpy is the state function, the enthalpy change $\Delta H_{\left(T_{1} \rightarrow T_{2}\right)}$ may be expressed also as

$$
\begin{aligned}
\Delta H_{\left(T_{1} \rightarrow T_{2}\right)}= & \int_{T_{1}}^{T_{\text {ref }}}\left(\bar{c}_{p_{2}(T)}\right)_{F} d T+\Delta H_{\left(T_{\text {ref }}\right)} \\
& +\int_{T_{\text {ref }}}^{T_{2}}\left(\bar{c}_{p_{2}(T)}\right)_{U} d T,
\end{aligned}
$$

where $\left(\bar{c}_{p_{2}(T)}\right)_{F}$ and $\left(\bar{c}_{p_{2}(T)}\right)_{U}$ are the partial molar heat capacities of the folded and unfolded DNA conformation, respectively, $\Delta H_{\left(T_{\text {ref }}\right)}$ is the enthalpy of unfolding at $T_{\text {ref }}$ which can be any temperature between $T_{1}$ and $T_{2}$. By choosing $T_{\text {ref }}=T_{1 / 2}$ where $T_{1 / 2}$ is the melting temperature at which a half of oligonucleotide molecules undergo unfolding transition (2) transforms into

$$
\begin{aligned}
\Delta H_{T_{(1 / 2)}}^{\mathrm{cal}}= & \int_{T_{1}}^{T_{1 / 2}}\left[\bar{c}_{p_{2}(T)}-\left(\bar{c}_{p_{2}(T)}\right)_{F}\right] d T \\
& +\int_{T_{1 / 2}}^{T_{2}}\left[\bar{c}_{p_{2}(T)}-\left(\bar{c}_{p_{2}(T)}\right)_{U}\right] d T,
\end{aligned}
$$




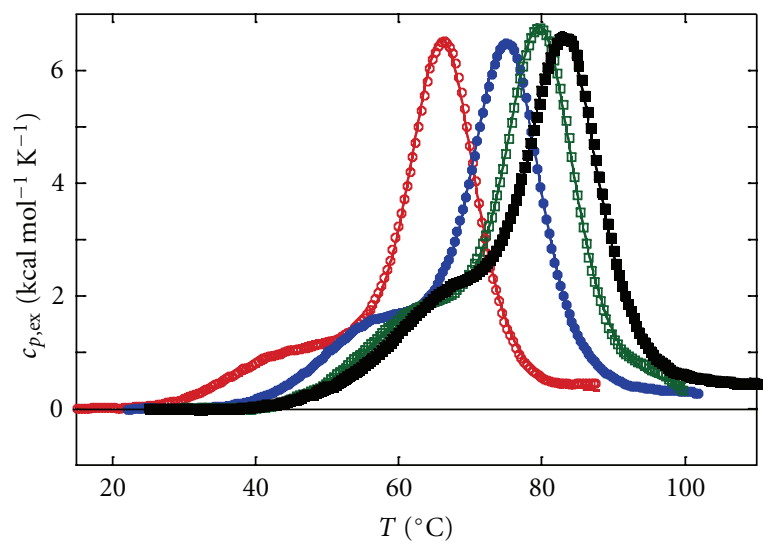

$\left[\mathrm{Na}^{+}\right](\mathrm{M})$

- 0.03

- 0.13

- 0.33

- 1.03

(a)

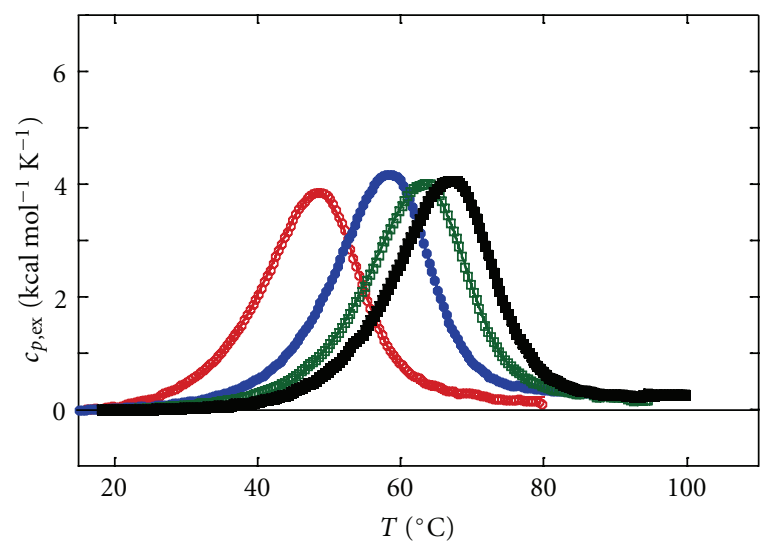

$\left[\mathrm{Na}^{+}\right](\mathrm{M})$

- 0.03

- 0.13

ㅁ 0.33

- 1.03

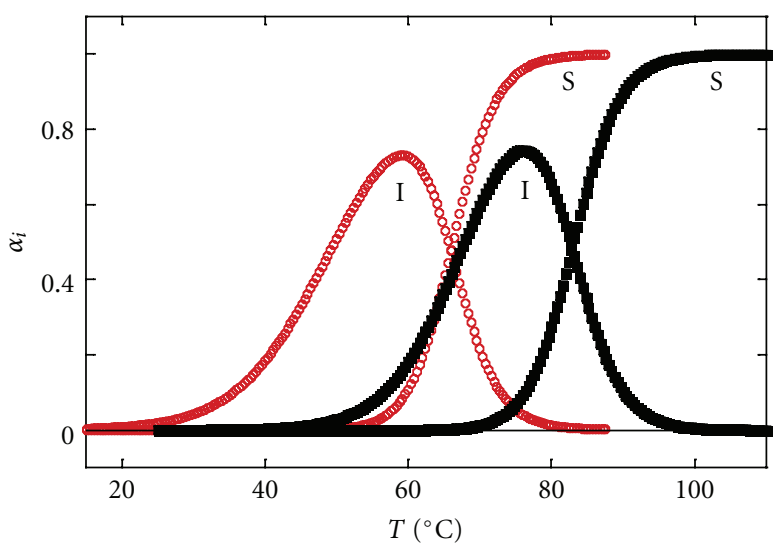

$\left[\mathrm{Na}^{+}\right](\mathrm{M})$

- 0.03

- 1.03 (b)

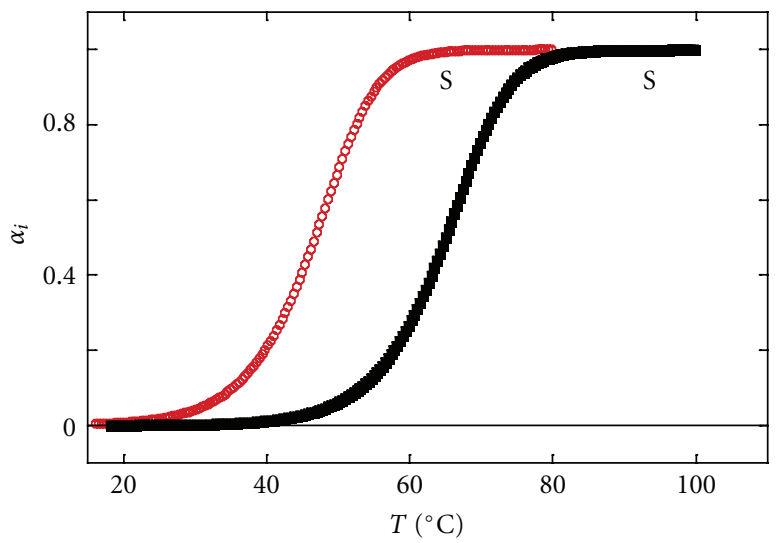

$\left[\mathrm{Na}^{+}\right](\mathrm{M})$

- 0.03

- 1.03

(c)

(d)

FIGURE 2: DSC thermograms and their model analysis: hairpin (HP) unfolding characterized in terms of a three-state model HP $\leftrightarrow$ I $\leftrightarrow$ $\mathrm{S}$ (a) and the corresponding fractions of species (b); duplex (D) unfolding characterized in terms of a two-state model D $\leftrightarrow 2 \mathrm{~S}$ (c) and the corresponding fractions of species (d). In panels (a) and (c) symbols represent experimental data points while lines of the same color correspond to the best-fit model functions ((14) and (18)).

where $\Delta H_{T_{1 / 2}}^{\text {cal }}$ is a model-independent enthalpy of transition at $T_{1 / 2}$ that can be easily determined by the appropriate integration of the experimental $\left[\bar{c}_{p_{2}(T)}-\left(\bar{c}_{p_{2}(T)}\right)_{F}\right]$ and $\left[\bar{c}_{p_{2}(T)}-\right.$ $\left.\left(\bar{c}_{p_{2}(T)}\right)_{U}\right]$ curves as presented in $(3)$.

According to the DSC thermograms of the measured hairpin (HP), its thermally induced unfolding involves at least two conformational transitions (Figure 2). Thus, the simplest suggested model to describe the observed thermal behavior would consist of two consecutive monomolecular transitions: HP (hairpin) $\leftrightarrow$ I (intermediate state) $\leftrightarrow$ S (unfolded single-stranded state). The enthalpy, $\mathrm{H}$, of a solution containing an HP sample characterized by the suggested thermal unfolding

$$
\mathrm{HP} \stackrel{K_{\mathrm{HPI}}}{\longleftrightarrow} \stackrel{K_{\mathrm{IS}}}{\longleftrightarrow} \mathrm{S} ; \quad K_{\mathrm{HPI}}=\frac{[\mathrm{I}]}{[\mathrm{HP}]} \quad K_{\mathrm{IS}}=\frac{[\mathrm{S}]}{[\mathrm{I}]}
$$

can be expressed at given $P$ and $T$ as

$$
H=n_{1} \bar{H}_{1}+n_{2} \bar{H}_{2}=n_{1} \bar{H}_{1}+n_{\mathrm{HP}} \bar{H}_{\mathrm{HP}}+n_{\mathrm{I}} \bar{H}_{\mathrm{I}}+n_{\mathrm{S}} \bar{H}_{\mathrm{S}},
$$


where $K_{\mathrm{HPI}}$ and $K_{\mathrm{IS}}$ are the corresponding equilibrium constants, the quantities in brackets are the equilibrium molar concentrations of HP, I, and S, $n_{1}$ is the number of moles of solvent, and $n_{2}$ is the number of moles of solute (oligonucleotide) that can be further expressed as:

$$
n_{2}=n_{\mathrm{HP}}+n_{\mathrm{I}}+n_{\mathrm{S}},
$$

$n_{\mathrm{HP}}$ in (6) represents the number of moles of the oligonucleotide in the folded hairpin state, $n_{\mathrm{I}}$ is the number of moles in the intermediate state, $n_{\mathrm{S}}$ is the number of moles in the unfolded single-stranded state and $\bar{H}_{1}, \bar{H}_{2}, \bar{H}_{\mathrm{HP}}, \bar{H}_{\mathrm{I}}$, and $\bar{H}_{\mathrm{S}}$ are the corresponding partial molar enthalpies of the solvent, solute and folded, intermediate and unfolded oligonucleotide, respectively. By defining the molar fraction, $\alpha_{i}$, of the solute species, $i$, as $\alpha_{i}=n_{i} / n_{2}$ one obtains from (5) that

$$
\bar{H}_{2}=\alpha_{\mathrm{HP}} \bar{H}_{\mathrm{HP}}+\alpha_{I} \bar{H}_{I}+\alpha_{\mathrm{S}} \bar{H}_{\mathrm{S}} .
$$

Finally, by introducing $\alpha_{\mathrm{HP}}=1-\alpha_{\mathrm{I}}-\alpha_{\mathrm{S}}$ into (7) and taking the temperature derivative of the modified (7) one obtains the model function for the measured DSC signal, $c_{P, \mathrm{ex}}$, expressed as

$$
\begin{aligned}
c_{P, \mathrm{ex}}= & \bar{c}_{P, 2}-\bar{c}_{P, \mathrm{HP}} \\
= & \alpha_{\mathrm{I}} \Delta c_{P, \mathrm{HPI}}+\frac{d \alpha_{\mathrm{I}}}{d T} \Delta H_{\mathrm{HPI}}+\alpha_{\mathrm{S}}\left(\Delta c_{P, \mathrm{HPI}}+\Delta c_{P, \mathrm{IS}}\right) \\
& +\frac{d \alpha_{\mathrm{S}}}{d T}\left(\Delta H_{\mathrm{HPI}}+\Delta H_{\mathrm{IS}}\right),
\end{aligned}
$$

in which at any temperature $\bar{c}_{P, 2}$ is the measured $c_{P}$ (with subtracted baseline), $\bar{c}_{P, \mathrm{HP}}$ is the partial molar heat capacity of HP extrapolated from low-temperature region over the entire measured temperature interval, $\Delta H_{\mathrm{HPI}}=\bar{H}_{\mathrm{I}}-\bar{H}_{\mathrm{HP}}$ (enthalpy of the hairpin to intermediate state transition), $\Delta H_{\mathrm{IS}}=\bar{H}_{\mathrm{S}}-\bar{H}_{\mathrm{I}}$ (enthalpy of the intermediate state to unfolded single stranded state transition), $\Delta c_{P, \mathrm{HPI}}=\bar{c}_{P, \mathrm{I}}-\bar{c}_{P, \mathrm{HP}}$ and $\Delta c_{P, \mathrm{IS}}=\bar{c}_{P, \mathrm{~S}}-\bar{c}_{P, \mathrm{I}}$.

$c_{P, \text { ex }}$ can be obtained experimentally simply by subtracting the hairpin $\bar{c}_{P, \mathrm{HP}}$ versus $T$ curve extrapolated from low$T$ region over the entire measured temperature interval from the corresponding measured $\bar{c}_{P, 2}$ versus $T$ curve. The modelbased $c_{P \text {,ex }}$, however, can be calculated from the right-hand side term of (8). According to the suggested model (4) the total solute molar concentration, $c_{T}$, and the fractions $\alpha_{i}$ of the solute species present in the solution can be expressed as $c_{T}=[\mathrm{HP}]+[\mathrm{I}]+[\mathrm{S}]$ and $\alpha_{\mathrm{HP}}=[\mathrm{HP}] / c_{T}, \alpha_{\mathrm{I}}=[\mathrm{I}] / c_{T}$ and $\alpha_{\mathrm{S}}=[\mathrm{S}] / c_{T}$. Since $\alpha_{\mathrm{HP}}+\alpha_{\mathrm{I}}+\alpha_{\mathrm{S}}=1$ one obtains from (4) that

$$
\alpha_{\mathrm{S}}=\frac{1}{\left(K_{\mathrm{HPI}} K_{\mathrm{IS}}\right)^{-1}+K_{\mathrm{S}}^{-1}+1}, \quad \alpha_{\mathrm{I}}=\frac{\alpha_{\mathrm{S}}}{K_{\mathrm{IS}}} .
$$

For the description of the DSC experiment with the model function (8) one needs also the temperature derivatives of $\alpha_{S}$ and $\alpha_{\mathrm{I}}$. By using for each transition, $i$, the van't Hoff relation

$$
\frac{d \ln K_{i}}{d T}=\frac{\Delta H_{i}^{0}}{R T^{2}}
$$

one obtains

$$
\begin{aligned}
& \frac{d \alpha_{\mathrm{S}}}{d T}=\alpha_{\mathrm{S}}^{2}\left[K_{\mathrm{HPI}}^{-1} K_{\mathrm{IS}}^{-1} \frac{\left(\Delta H_{\mathrm{HPI}}^{0}+\Delta H_{\mathrm{IS}}^{0}\right)}{R T^{2}}+K_{\mathrm{IS}}^{-1} \frac{\Delta H_{\mathrm{IS}}^{0}}{R T^{2}}\right], \\
& \frac{d \alpha_{\mathrm{I}}}{d T}=K_{\mathrm{IS}}^{-1}\left(\frac{d \alpha_{\mathrm{S}}}{d T}-\alpha_{\mathrm{S}} \frac{\Delta H_{\mathrm{IS}}^{0}}{R T^{2}}\right) .
\end{aligned}
$$

Assuming that for each transition, $i$, the corresponding $\Delta c_{P_{i}}^{0}$ does not depend on $T$ the standard free energy of that transition, $\Delta G_{i(T)}^{0}$, can be obtained at any $T$ from the integrated form of the Gibbs-Helmholtz relation as

$$
\begin{aligned}
\Delta G_{i(T)}^{0}=T & {\left[\frac{\Delta G_{i\left(T_{i,(1 / 2)}\right)}^{0}}{T_{i, 1 / 2}}+\Delta H_{i\left(T_{i, 1 / 2}\right)}^{0}\left(\frac{1}{T}-\frac{1}{T_{i, 1 / 2}}\right)\right.} \\
& \left.+\Delta c_{P_{i}}^{0}\left(1-\frac{T_{i, 1 / 2}}{T}-\ln \frac{T}{T_{i, 1 / 2}}\right)\right],
\end{aligned}
$$

where $T_{i, 1 / 2}$ is the temperature at which the $\alpha_{i}$ values sof species participating in transition $i$ are the same. The corresponding equilibrium constant, $K_{i}$, is related to $\Delta G_{i(T)}^{0}$ as

$$
\Delta G_{i(T)}^{0}=-R T \ln K_{i},
$$

and for the suggested mechanism of the hairpin unfolding (4) it can be easily seen that for each suggested monomolecular transition $\Delta G_{i\left(T_{i, 1 / 2}\right)}^{0}=0$. Finally, according to the DSC experiments performed at different oligonucleotide concentrations the $\Delta H_{i(T)}$ values appear to be concentration independent thus indicating that one may assume for each transition that $\Delta H_{i(T)}=\Delta H_{i(T)}^{0}$ and $\Delta c_{P_{i}}=\Delta c_{P_{i}}^{0}$. Using these assumptions and (8)-(13) one can express the model function (14)

$$
\begin{aligned}
c_{P, \mathrm{ex}}= & \alpha_{\mathrm{I}} \Delta c_{p, \mathrm{HPI}}^{0}+\frac{d \alpha_{\mathrm{I}}}{d T} \Delta H_{\mathrm{HPI}}^{0}+\alpha_{\mathrm{S}}\left(\Delta c_{p, \mathrm{HPI}}^{0}+\Delta c_{p, \mathrm{IS}}^{0}\right) \\
& +\frac{d \alpha_{\mathrm{S}}}{d T}\left(\Delta H_{\mathrm{HPI}}^{0}+\Delta H_{\mathrm{IS}}^{0}\right),
\end{aligned}
$$

only in terms of parameters $T_{i, 1 / 2}, \Delta H_{i\left(T_{i, 1 / 2}\right)}^{0}$, and $\Delta c_{P, i}^{0}$, characteristic for each of the suggested transitions. Their "best fit" values are obtained by fitting the model function (14) to the experimental $c_{P, \text { ex }}$ versus $T$ curves. Furthermore, since for each transition, $i$, the corresponding $\Delta H_{i(T)}^{0}$ and $\Delta S_{i(T)}^{0}$ quantities can be expressed as

$$
\begin{gathered}
\Delta H_{i(T)}^{0}=\Delta H_{i\left(T_{i, 1 / 2}\right)}^{0}+\Delta c_{P, i}^{0}\left(T-T_{i, 1 / 2}\right), \\
T \Delta S_{i(T)}^{0}=\Delta H_{i(T)}^{0}-\Delta G_{i(T)}^{0},
\end{gathered}
$$

the "best fit" parameters $T_{i, 1 / 2}, \Delta H_{i\left(T_{i, 1 / 2}\right)}^{0}$ and $\Delta c_{P, i}^{0}$ can be used also to obtain the $\Delta H_{i(T)}^{0}$ and $\Delta S_{i(T)}^{0}$ values at any $T$.

In contrast to HP unfolding, the measured thermally induced duplex (D) to single strand (S) transition appears to be a simpler, all-or-none process

$$
D \stackrel{K_{\mathrm{DS}}}{\longleftrightarrow} 2 \mathrm{~S} ; \quad K_{\mathrm{DS}}=\frac{[\mathrm{S}]^{2}}{[\mathrm{D}]},
$$


that can be described in terms of the total oligonucleotide concentration, $c_{T}$, the concentrations of the duplex form [D] and the single strands [S], the fraction of duplex molecules that undergo the unfolding transition at a given temperature, $\alpha_{\mathrm{S}}$, and the equilibrium constant $K_{\mathrm{DS}}$ interrelated as

$$
c_{T}=[\mathrm{D}]+\frac{[\mathrm{S}]}{2} ; \quad \alpha_{\mathrm{S}}=\frac{[\mathrm{D}]}{c_{T}} ; \quad K_{\mathrm{DS}}=\frac{4 \alpha_{\mathrm{S}}^{2} c_{T}}{1-\alpha_{\mathrm{S}}} .
$$

A similar, though much simpler derivation of the model function than the one presented for unfolding of the hairpin structure (14) leads for the suggested $\mathrm{D} \leftrightarrow 2 \mathrm{~S}$ transition to

$$
c_{P, \mathrm{ex}}=\bar{c}_{P, 2}-\bar{c}_{P, \mathrm{D}}=\alpha_{\mathrm{S}} \Delta c_{P, \mathrm{DS}}^{0}+\frac{d \alpha_{\mathrm{S}}}{d T} \Delta H_{\mathrm{DS}}^{0},
$$

where $\bar{c}_{P, 2}$ is the measured $c_{P}$ of the sample solution with subtracted baseline, $\bar{c}_{P, \mathrm{D}}$ is the heat capacity of the duplex form extrapolated from the low- $T$ region over the whole measured temperature interval, $\Delta c_{P, \mathrm{DS}}^{0}=\Delta c_{P, \mathrm{DS}}=2 \bar{c}_{P_{\mathrm{S}}}-\bar{c}_{P_{\mathrm{D}}}$ and $\Delta H_{\mathrm{DS}}^{0}=\Delta H_{\mathrm{DS}}=2 \bar{H}_{\mathrm{S}}-\bar{H}_{\mathrm{D}}$. From the suggested model (16) and (17) it follows that $\Delta G_{\mathrm{DS}\left(T_{1 / 2}\right)}^{0}=-R T \ln \left(2 c_{T}\right)$ and

$$
\begin{gathered}
\alpha_{\mathrm{S}}=\frac{1}{2}\left[-\frac{K_{\mathrm{DS}}}{4 c_{T}}+\sqrt{\left(\frac{K_{\mathrm{DS}}}{4 c_{T}}\right)^{2}+\frac{K_{\mathrm{DS}}}{c_{T}}}\right], \\
\frac{\partial \alpha_{\mathrm{S}}}{\partial T}=\frac{\Delta H_{\mathrm{DS}}^{0}}{R T^{2}} \frac{\alpha_{\mathrm{S}}\left(1-\alpha_{\mathrm{S}}\right)}{2-\alpha_{\mathrm{S}}} .
\end{gathered}
$$

The corresponding expressions for $\Delta G_{\mathrm{DS}(T)}^{0} \Delta H_{\mathrm{DS}(T)}^{0}$ and $T \Delta S_{\mathrm{DS}(T)}^{0}$ are the same as those shown for each transition in the suggested hairpin unfolding mechanism (12), (13) and (15). Similarly, in deriving (18) the $\Delta H_{\mathrm{DS}(T)}$ and $\Delta c_{P, \mathrm{DS}}$ are assumed to be independent on the oligonucleotide concentration and thus equal to $\Delta H_{\mathrm{DS}(T)}^{0}$, and $\Delta c_{P, \mathrm{DS}}^{0}$.

Inspection of (18) and (19) shows that the model function (18) is expressed in terms of adjustable parameters, $T_{1 / 2}$, $\Delta H_{\mathrm{DS}\left(T_{1 / 2}\right)}^{0}$ and $\Delta c_{P, \mathrm{DS}}^{0}$ that can be determined by fitting the model function to the experimental $c_{P, \text { ex }}$ versus $T$ curve and further used to determine the $\Delta G_{\mathrm{DS}(T)}^{0}, \Delta H_{\mathrm{DS}(T)}^{0}$ and $\Delta S_{\mathrm{DS}(T)}^{0}$ values at any $T$.

To obtain a set of the "best fit" adjustable parameters $T_{i, 1 / 2}, \Delta H_{i\left(T_{i, 1 / 2}\right)}^{0}$ and $\Delta c_{P, i}^{0}$ describing the hairpin and duplex thermal unfolding at each of the added salt concentrations the iterative nonlinear Levenberg-Marquardt $\chi^{2}$ regression procedure was used [31]. Furthermore, assuming that for the observed transitions the accompanying $\Delta c_{P, i}^{0}$ quantities do not depend on the added $\mathrm{NaCl}$ concentration their values may be determined also from the slopes of the $\Delta H_{i\left(T_{1 / 2}\right)}^{0}$ versus $T_{i, 1 / 2}$ curves constructed from the "best fit" $\Delta H_{i\left(T_{1 / 2}\right)}^{0}$ and $T_{i, 1 / 2}$ parameters determined at different added salt concentrations [32]. These data can be also used to construct the corresponding $T_{i, 1 / 2}$ versus $\ln \left[\mathrm{Na}^{+}\right]$plots from which the amount of the $\mathrm{Na}^{+}$ions released upon thermal unfolding of the hairpin or duplex structure can be estimated (see discussion, (20)).

\section{Results and Discussion}

According to the measured DSC thermograms presented in Figure 2 the thermally induced unfolding of the hairpin HP consists of at least two conformational transitions while the one observed for the duplex D occurs in a simpler "all or none" manner. In addition, UV melting experiments (not shown) resulted for HP in biphasic melting curves that exhibit transitions independent on HP concentration (monomolecular transitions) while for D monophasic melting curves dependent on $\mathrm{D}$ concentration (nonmonomolecular transition) were observed. Moreover, excellent repeatability of the consecutive measured heating and cooling $c_{P}$ versus $T$ curves and the observed independence of the measured DSC peaks on the applied heating rate (several test experiments) clearly shows that the studied thermal unfolding events may be considered as reversible processes. Model analysis of the measured thermograms shows that the hairpin thermal unfolding can be well described in terms of a three state model involving $\mathrm{H}$ (hairpin) $\longleftrightarrow \mathrm{I}$ (intermediate state) $\longleftrightarrow$ $S$ (unfolded single-stranded structure) transitions and the corresponding model function (14) characterized for each of the suggested transitions with the corresponding "best fit" adjustable parameters $T_{i, 1 / 2}, \Delta H_{i\left(T_{i, 1 / 2}\right)}^{0}$, and $\Delta c_{P, i}^{0}$ (Table 1). However, analysis of the applied fitting procedure indicates that the parameter $\Delta c_{P, I S}^{0}$ is highly correlated to some other adjustable parameters. Thus, to obtain safe estimate of $\Delta c_{P, I S}^{0}$ another method of its determination has to be used. Assuming that it does not depend on the simple salt concentration $\Delta c_{P, I S}^{0}$ was estimated as a slope of the $\Delta H_{I S\left(T_{1 / 2}\right)}^{0}$ versus $T_{I S, 1 / 2}$ plot (Figure 3(a)) constructed from the "best fit" parameters determined at different $\mathrm{NaCl}$ concentrations (Table 1). This method of determining $\Delta c_{P, i}^{0}$ was justified by a good agreement between the $\Delta c_{P, i}^{0}$ values for other transitions obtained by the described fitting procedure and the $\Delta c_{P_{i}}^{0}$ values determined as the slopes of the corresponding $\Delta H_{i\left(T_{i, 1 / 2}\right)}^{0}$ versus $T_{i, 1 / 2}$ plots (Table 1 ). By using the parameters presented in Table 1 one can calculate for the duplex and hairpin solutions the relative populations of the modelpredicted species in the measured temperature interval and at all added salt concentrations (Figure 2). Evidently, the thermal stability of the folded state of the measured duplex and the hairpin is substantially enhanced by increasing the added salt concentration. At low salt concentrations, however, a small fraction of the hairpin molecules undergoes transition into the intermediate state already at physiological temperatures.

A standard way of testing the quality of a suggested model is to compare the enthalpy of unfolding determined at a given temperature directly by an appropriate integration of the experimental $c_{P, \text { ex }(T)}$ versus $T$ curve $\left(\Delta H_{H S}^{\mathrm{cal}}\right.$, see (3)) with the corresponding model-based value $\Delta H_{\mathrm{HS}}^{0}$ calculated at the same temperature using the reported "best fit" adjustable parameters. As shown in Table 1 a good agreement was obtained which clearly supports the appropriateness of the suggested $H \longleftrightarrow I \longleftrightarrow S$ unfolding model.

It is well known that DNA unfolding is accompanied by release of counterions. The number of the released $\mathrm{Na}^{+}$ions, 
TABLE 1: Thermodynamic parameters ${ }^{\mathrm{a}}$ obtained from fitting the model functions ((14) and (18)) to the duplex (D) and hairpin (HP) DSC thermograms presented in Figure 2.

\begin{tabular}{|c|c|c|c|c|c|c|}
\hline Transition & $T_{1 / 2}$ & $\Delta H_{\left(T_{1 / 2}\right)}^{0}$ & $\Delta H_{\left(T_{1 / 2}\right)}^{\mathrm{cal}}$ & $\Delta c_{P}^{0 \mathrm{~b}}$ & $\Delta c_{P}^{0} \mathrm{c}$ & $\Delta n_{\mathrm{Na}^{+}}$ \\
\hline $\mathrm{HP} \rightarrow \mathrm{I}$ & 59.2 & 37 & & & 0.34 & 0.9 \\
\hline $\mathrm{I} \rightarrow \mathrm{S}$ & 75.0 & 74 & & 0.37 & 0.25 & 1.5 \\
\hline $\mathrm{HP} \rightarrow \mathrm{S}$ & & $117^{\mathrm{d}}$ & 111 & & 0.59 & 2.4 \\
\hline $\mathrm{D} \rightarrow 2 \mathrm{~S}$ & 56.5 & 71 & 71 & 0.25 & 0.30 & 1.7 \\
\hline Error & \pm 0.2 & \pm 2 & \pm 2 & \pm 0.05 & \pm 0.05 & \pm 0.2 \\
\hline
\end{tabular}

${ }^{a}$ Units: ${ }^{\circ} \mathrm{C}\left(T_{1 / 2}\right), \mathrm{kcal} \mathrm{mol}^{-1}\left(\Delta H_{\left(T_{1 / 2}\right)}^{0}, \Delta H_{\left(T_{1 / 2}\right)}^{\mathrm{cal}}\right), \mathrm{kcal} \mathrm{mol}{ }^{-1} \mathrm{~K}^{-1}\left(\Delta c_{P}^{0}\right)$; unless stated otherwise the values are those obtained at $\left[\mathrm{Na}^{+}\right]=0.13 \mathrm{M}$;

bobtained from fitting the model function;

c obtained as the slope of $\Delta H_{\left(T_{1 / 2}\right)}^{0}$ versus $T_{1 / 2}$ curves (Figure 3(a));

$\mathrm{d}$ the total enthalpy of the of the I $\rightarrow \mathrm{S}$ transition was calculated as $\Delta H_{\mathrm{HS}\left(T_{1 / 2}, \mathrm{IS}\right)}^{0}=\Delta H_{\mathrm{HI}\left(T_{1 / 2}, \mathrm{HI}\right)}^{0}+\Delta c_{P_{\mathrm{HI}}}^{0}\left(T_{1 / 2, \mathrm{IS}}-T_{1 / 2, \mathrm{HI}}\right)+\Delta H_{\mathrm{IS}\left(T_{1 / 2}, \mathrm{IS}\right)}^{0}$ where $T_{1 / 2, \mathrm{IS}}$ and $T_{1 / 2, \mathrm{HI}}$ are the melting temperatures of the $\mathrm{I} \rightarrow \mathrm{S}$ and $\mathrm{H} \rightarrow \mathrm{I}$ transitions and $\Delta H_{\mathrm{IS}\left(T_{1 / 2}, \mathrm{IS}\right)}^{0}$ and $\Delta H_{\mathrm{HI}\left(T_{1 / 2}, \mathrm{HI}\right)}^{0}$ are the corresponding enthalpies of transition.

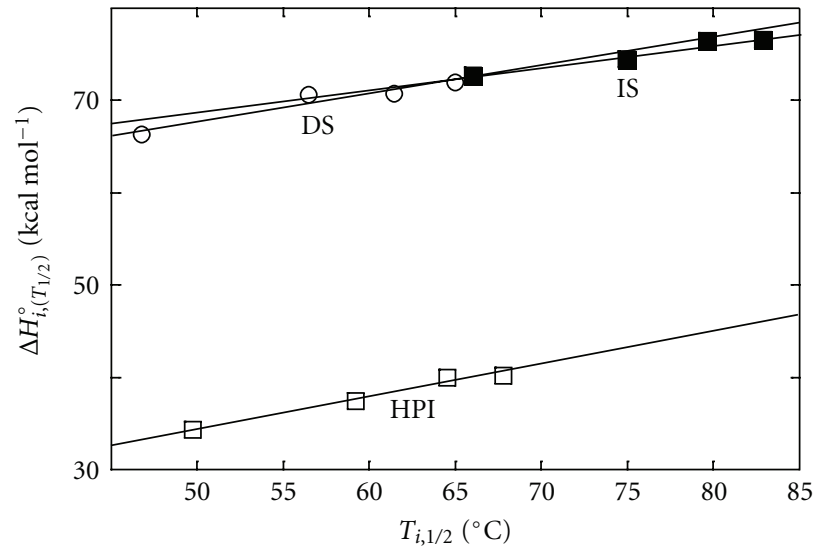

(a)

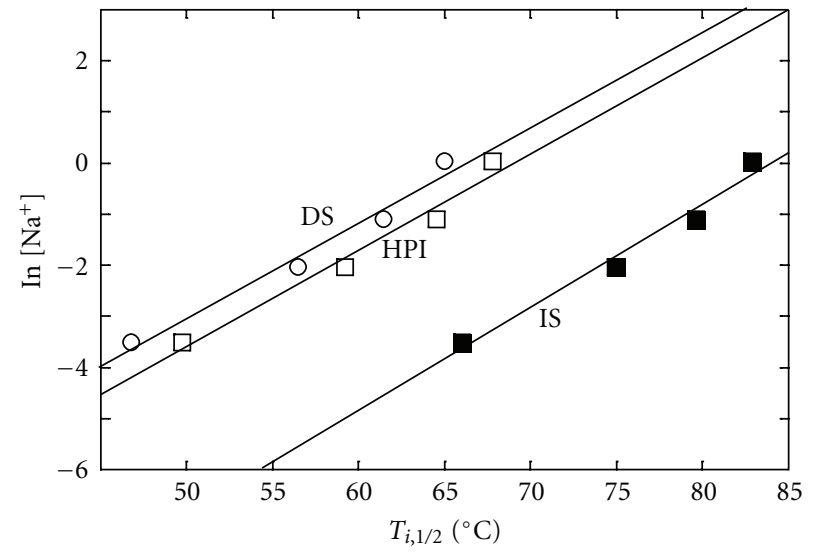

(b)

FIgURE 3: Estimation of heat capacity changes and number of released $\mathrm{Na}^{+}$ions: (a) $\Delta c_{P, i}^{0}$ was determined for each transition $i$ as the slope of the $\Delta H_{i\left(T_{i, 1 / 2}\right)}^{0}$ versus $T_{i, 1 / 2}$ plot constructed from the model based $\Delta H_{i\left(T_{i, 1 / 2}\right)}^{0}$ and $T_{i, 1 / 2}$ values determined at different salt concentrations; (b) the corresponding $\ln \left[\mathrm{Na}^{+}\right]$versus $T_{i, 1 / 2}$ plots from which the $\Delta n_{\mathrm{Na}^{+}, i}$ values were determined according to (20).

$\Delta n_{\mathrm{Na}^{+}, i}$, upon each HP and D transition, expressed per mole of oligonucleotide, may be estimated from [33]

$$
\frac{d T_{i, 1 / 2}}{d \ln \left[\mathrm{Na}^{+}\right]}=\frac{R T_{i, 1 / 2}^{2}}{\Delta H_{i\left(T_{i, 1 / 2}\right)}^{0}} \Delta n_{\mathrm{Na}^{+}, i}
$$

in which $T_{i, 1 / 2}$ is the melting temperature at a given $\mathrm{Na}^{+}$concentration, $\left[\mathrm{Na}^{+}\right], \Delta H_{i\left(T_{i, 1 / 2}\right)}^{0}$ is the corresponding enthalpy of transition at $T_{i, 1 / 2}$. The $\Delta n_{N a^{+}, i}$ values presented in Table 1 were determined from the slopes of the $\ln \left[\mathrm{Na}^{+}\right]$ versus $T_{i, 1 / 2}$. plots (Figure $3(\mathrm{~b})$ ).

At any $T$ in the measured range of physiological temperatures the difference between the given property characterizing the total unfolding of the hairpin $\mathrm{H}$ and the duplex D (for ex. $\Delta \Delta H_{(T)}^{0}=\Delta H_{\mathrm{HS}(T)}^{0}-\Delta H_{\mathrm{DS}(T)}^{0}$ ) reflects the contribution of the TCTC loop to that property relative to the core duplex (Figure 4, Table 2). Thus, the observed $\Delta \Delta H_{(T)}^{0}>0$ indicates a favorable energy contribution of the TCTC loop to the stability of the hairpin that results, very likely, from the increased number of stacking interactions (in the first place from those occurring at the core duplexloop connections) [34]. The corresponding $\Delta \Delta S_{(T)}^{0}>0$ is consistent with the highly positive $\Delta \Delta H_{(T)}^{0}$ indicating that the unfavorable entropy contribution of the TCTC loop to the hairpin stability arises largely from a substantial disruption of the loop structure accompanying the unfolding of the hairpin. The observed $\Delta \Delta c_{P}^{0}>0$ and $\Delta \Delta n_{\mathrm{Na}^{+}}>0$ show, however, that the loop contributions to $\Delta \Delta H_{(T)}^{0}$ and $\Delta \Delta S_{(T)}^{0}$ may be, to a certain extent, determined also by hydration [35] and electrostatic interactions. The $\Delta \Delta c_{P}^{0}>0$ suggests that within the folded hairpin conformation, not only the core duplex but also the TCTC loop are less exposed to water than in the unfolded state. In addition, the observed $\Delta \Delta n_{\mathrm{Na}^{+}}>0$ may be ascribed to a decrease in the surface charge density accompanying the unfolding of the oligonucleotide which is significantly more pronounced in the case of hairpin unfolding. Comparison of the $\Delta \Delta$ values for $\Delta G_{T}^{0}, \Delta H_{T}^{0}, \Delta S_{T}^{0}, \Delta c_{P}^{0}$, and $\Delta n_{\mathrm{Na}^{+}}$quantities determined for the HP $\rightarrow$ I and I $\rightarrow \mathrm{S}$ transitions of the hairpin with the corresponding $\Delta \Delta$ values determined for the $\mathrm{D} \rightarrow 2 \mathrm{~S}$ transition of the duplex shows that the $\Delta \Delta$ values for the $\mathrm{I} \rightarrow \mathrm{S}$ and $\mathrm{D} \rightarrow 2 \mathrm{~S}$ transitions are very close (Figure 4 , Table 2 ). Evidently, one may speculate that in the hairpin structure only the HP $\rightarrow$ I transition is influenced by the TCTC loop. In other words, it seems 
TABLE 2: Difference thermodynamic stability parameters ${ }^{\mathrm{a}}$ at $25^{\circ} \mathrm{C}$ exhibiting the influence of the TCTC loop to the unfolding features of the hairpin forming oligonucleotide (HP).

\begin{tabular}{lccccc}
\hline Transition & $\Delta \Delta G^{0}$ & $\Delta \Delta H^{0}$ & $T \Delta \Delta S^{0}$ & $\Delta \Delta c_{P}^{0}$ & $\Delta \Delta n_{N a^{+}}$ \\
\hline$(\mathrm{HP} \rightarrow \mathrm{S})-(\mathrm{D} \rightarrow 2 \mathrm{~S})$ & 2.4 & 26.6 & 24.3 & 0.29 & 0.7 \\
$(\mathrm{I} \rightarrow \mathrm{S})-(\mathrm{D} \rightarrow 2 \mathrm{~S})$ & -0.9 & 0.8 & 1.7 & -0.05 & -0.2 \\
\hline Error & \pm 3 & \pm 3 & \pm 3 & \pm 0.07 & \pm 0.3 \\
\hline
\end{tabular}

${ }^{\mathrm{a}}$ Units: kcal mol ${ }^{-1}\left(\Delta \Delta G^{0}, \Delta \Delta H^{0}, T \Delta \Delta S^{0}\right), \mathrm{kcal} \mathrm{mol}^{-1} \mathrm{~K}^{-1}\left(\Delta \Delta c_{P}^{0}\right)$; for temperature dependence of $\Delta G^{0}, \Delta H^{0}$, and $T \Delta S^{0}$ see Figure 4 .

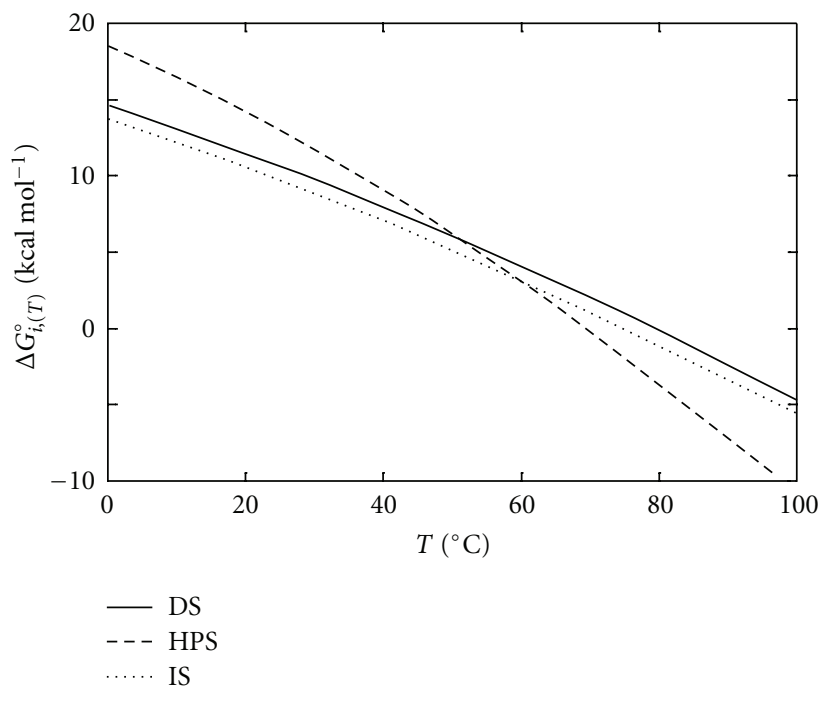

(a)

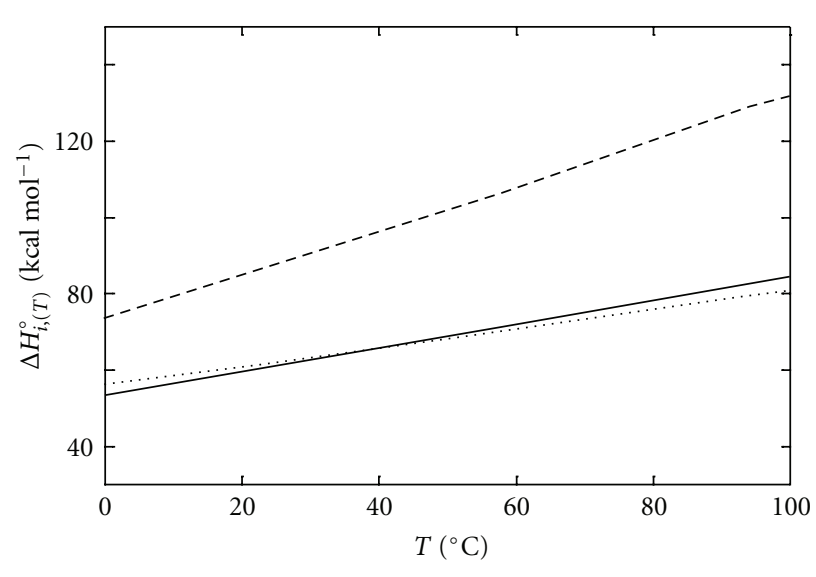

- DS

--- HPS

..... IS

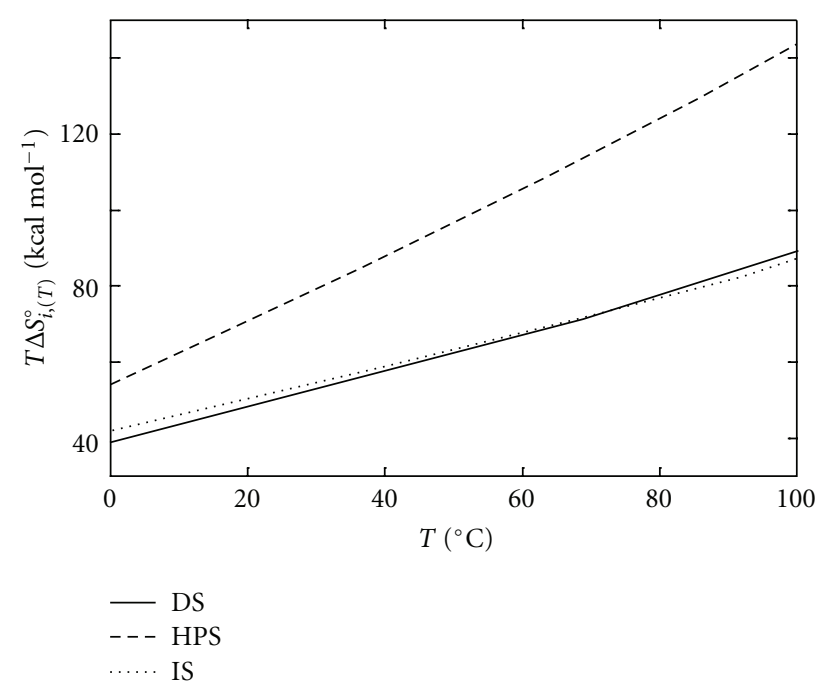

(c)

FIGURE 4: Thermodynamics of hairpin and duplex unfolding: standard Gibbs free energy (a), standard enthalpy (b), and the corresponding entropy contribution $(c)$ presented for each model predicted hairpin $(\mathrm{I} \rightarrow \mathrm{S}, \mathrm{HP} \rightarrow \mathrm{S})$ and duplex $(\mathrm{D} \rightarrow 2 \mathrm{~S})$ transitions as functions of temperature at $\left[\mathrm{Na}^{+}\right]=0.13 \mathrm{M}$.

that the observed HP $\rightarrow$ I transition reflects mainly the changes in the TCTC conformation. Thus, the intermediate state I may be considered as a state in which the core duplex remains more or less unchanged while the TCTC loop occurs as a more flexible structure characterized by the additional stacking interactions and the freedom of the neighboring water molecules and ions similar to the one in the unfolded state.

To the best of our knowledge this is the first time that a three-state unfolding of a simple hairpin structure, observed 
by DSC, has been reported and characterized thermodynamically. We believe that the main reason for this is that in most studies of thermal unfolding of hairpins too high starting temperatures have been chosen and therefore the low-temperature transitions have been overlooked.

\section{Acknowledgments}

This paper was supported by the Slovenian Research Agency through Grant no. P1-0201 and by the COST action MP0802.

\section{References}

[1] P. Brion and E. Westhof, "Hierarchy and dynamics of RNA folding," Annual Review of Biophysics and Biomolecular Structure, vol. 26, pp. 113-137, 1997.

[2] D. B. Roth, J. P. Menetski, P. B. Nakajima, M. J. Bosma, and M. Gellert, "V(D)J recombination: broken DNA molecules with covalently sealed (hairpin) coding ends in scid mouse thymocytes," Cell, vol. 70, no. 6, pp. 983-991, 1992.

[3] A. Bhasin, I. Y. Goryshin, and W. S. Reznikoff, "Hairpin formation in Tn5 transposition," Journal of Biological Chemistry, vol. 274, no. 52, pp. 37021-37029, 1999.

[4] A. K. Kennedy, A. Guhathakurta, N. Kleckner, and D. B. Haniford, "Tn10 transposition via a DNA hairpin intermediate," Cell, vol. 95, no. 1, pp. 125-134, 1998.

[5] O. C. Uhlenbeck, "Nucleic-acid structure-tetraloops and RNA folding," Nature, vol. 346, no. 6285, pp. 613-614, 1990.

[6] C. Cheong, G. Varani, and I. Tinoco, "Solution structure of an unusually stable RNA heparin-GGAC(UUCG)GUCC," Nature, vol. 346, no. 6285, pp. 680-682, 1990.

[7] H. A. Heus and A. Pardi, "Structural features that give rise to the unusual stability of RNA hairpins containing GNRA loops," Science, vol. 253, no. 5016, pp. 191-194, 1991.

[8] D. E. Draper, “Themes in RNA-protein recognition," Journal of Molecular Biology, vol. 293, no. 2, pp. 255-270, 1999.

[9] P. Svoboda and A. Di Cara, "Hairpin RNA: a secondary structure of primary importance," Cellular and Molecular Life Sciences, vol. 63, no. 7-8, pp. 901-918, 2006.

[10] G. Bonnet, S. Tyagi, A. Libchaber, and F. R. Kramer, "Thermodynamic basis of the enhanced specificity of structured DNA probes," Proceedings of the National Academy of Sciences of the United States of America, vol. 96, no. 11, pp. 6171-6176, 1999.

[11] Y. Kim, D. Sohn, and W. Tan, "Molecular beacons in biomedical detection and clinical diagnosis," International Journal of Clinical and Experimental Pathology, vol. 1, pp. 105-116, 2008.

[12] I. E. Scheffler, E. L. Elson, and R. L. Baldwin, "Helix formation by d(TA) oligomers. II. Analysis of the helix-coil transitions of linear and circular oligomers," Journal of Molecular Biology, vol. 48, no. 1, pp. 145-171, 1970.

[13] J. Gralla and D. M. Crothers, "Free energy of imperfect nucleic acid helices. II. Small Hairpin Loops," Journal of Molecular Biology, vol. 73, no. 4, pp. 497-511, 1973.

[14] C. A. Haasnoot, C. W. Hilbers, G. A. van der Marel et al., "On loop folding in nucleic acid hairpin-type structures," Journal of Biomolecular Structure \& Dynamics, vol. 3, no. 5, pp. 843-857, 1986.

[15] M. M. Senior, R. A. Jones, and K. J. Breslauer, "Influence of loop residues on the relative stabilities of DNA hairpin structures," Proceedings of the National Academy of Sciences of the United States of America, vol. 85, no. 17, pp. 6242-6246, 1988.

[16] M. Zuker, "On findings all suboptimal foldings of an RNA molecule," Science, vol. 244, no. 4900, pp. 48-52, 1989.

[17] D. Rentzeperis, K. Alessi, and L. A. Marky, "Thermodynamics of DNA hairpins: contribution of loop size to hairpin stability and ethidium binding," Nucleic Acids Research, vol. 21, no. 11, pp. 2683-2689, 1993.

[18] M. W. Freyer, R. Buscaglia, B. Nguyen, W. David Wilson, and E. A. Lewis, "Binding of netropsin and 4,6-diamidino2-phenylindole to an A2T2 DNA hairpin: a comparison of biophysical techniques," Analytical Biochemistry, vol. 355, no. 2, pp. 259-266, 2006.

[19] S. V. Kuznetsov, C. C. Ren, S. A. Woodson, and A. Ansari, "Loop dependence of the stability and dynamics of nucleic acid hairpins," Nucleic Acids Research, vol. 36, no. 4, pp. 10981112, 2008.

[20] J. Lah, I. Drobnak, M. Dolinar, and G. Vesnaver, "What drives the binding of minor groove-directed ligands to DNA hairpins?" Nucleic Acids Research, vol. 36, no. 3, pp. 897-904, 2008.

[21] J. Lah, N. Carl, I. Drobnak, B. Šumiga, and G. Vesnaver, "Competition of some minor groove binders for a single DNA binding site," Acta Chimica Slovenica, vol. 53, no. 3, pp. 284291, 2006.

[22] P. C. Bevilacqua and J. M. Blose, "Structures, kinetics, thermodynamics, and biological functions of RNA hairpins," Annual Review of Physical Chemistry, vol. 59, pp. 79-103, 2008.

[23] D. Bikard, C. Loot, Z. Baharoglu, and D. Mazel, "Folded DNA in action: hairpin formation and biological functions in prokaryotes," Microbiology and Molecular Biology Reviews, vol. 74, no. 4, pp. 570-588, 2010.

[24] L. A. Marky, D. Rentzeperis, and J. Ho, "Contribution of loops and nicks to the formation of DNA dumbbells: melting behavior and ligand binding," Biochemistry, vol. 32, no. 10, pp. 2564-2572, 1993.

[25] I. Prislan, J. Lah, and G. Vesnaver, "Diverse polymorphism of G-quadruplexes as a kinetic phenomenon," Journal of the American Chemical Society, vol. 130, no. 43, pp. 14161-14169, 2008.

[26] I. Prislan, J. Lah, M. Milanic et al., "Kinetically governed polymorphism of d(G4T4G3) quadruplexes in K+ solutions," Nucleic Acids Research, vol. 39, pp. 1933-1942, 2011.

[27] A. Ansari, S. V. Kuznetsov, and Y. Shen, "Configurational diffusion down a folding funnel describes the dynamics of DNA hairpins," Proceedings of the National Academy of Sciences of the United States of America, vol. 98, no. 14, pp. 7771-7776, 2001.

[28] Y. Shen, S. V. Kuznetsov, and A. Ansari, "Loop dependence of the dynamics of DNA hairpins," Journal of Physical Chemistry $B$, vol. 105, no. 48, pp. 12202-12211, 2001.

[29] H. Ma, D. J. Proctor, E. Kierzek, R. Kierzek, P. C. Bevilacqua, and M. Gruebele, "Exploring the energy landscape of a small RNA hairpin," Journal of the American Chemical Society, vol. 128, no. 5, pp. 1523-1530, 2006.

[30] C. R. Cantor, M. M. Warshaw, and H. Shapiro, "Oligonucleotide interactions. 3. Circular dichroism studies of the conformation of deoxyoligonucleotides," Biopolymers, vol. 9, no. 9, pp. 1059-1077, 1970.

[31] W. H. Press, B. P. Flannery, S. A. Teukolsky et al., Numerical Recipies, Cambridge University Press, Oxford, UK, 2007.

[32] I. Drobnak, M. Seručnik, J. Lah, and G. Vesnaver, "Stability of a short DNA duplex as a function of temperature: the effect of $\Delta \mathrm{Cp}$ and added salt concentration," Acta Chimica Slovenica, vol. 54, no. 3, pp. 445-451, 2007. 
[33] M. T. Record, C. F. Anderson, and T. M. Lohman, "Thermodynamic analysis of ion effects on the binding and conformational equilibria of proteins and nucleic acids: the roles of ion association or release, screening, and ion effects on water activity," Quarterly Reviews of Biophysics, vol. 11, no. 2, pp. 103-178, 1978.

[34] C. W. Hilbers, C. A. G. Haasnoot, S. H. de Bruin, J. J. M. Joordens, G. A. Van Der Marel, and J. H. Van Boom, "Hairpin formation in synthetic oligonucleotides," Biochimie, vol. 67, no. 7-8, pp. 685-695, 1985.

[35] P. M. Vallone and A. S. Benight, "Melting studies of short DNA hairpins containing the universal base 5-nitroindole," Nucleic Acids Research, vol. 27, pp. 3589-3596, 1999. 

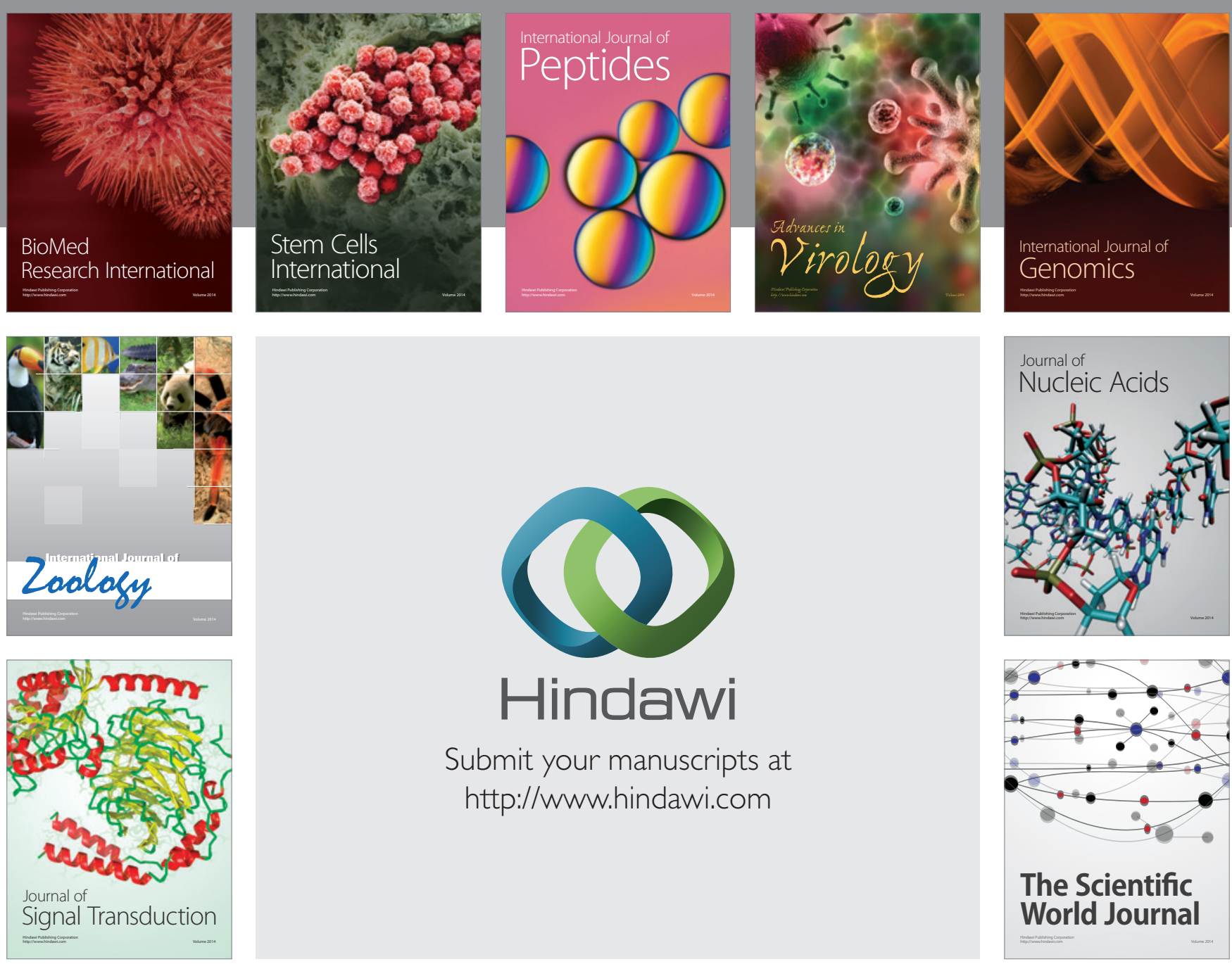

Submit your manuscripts at

http://www.hindawi.com
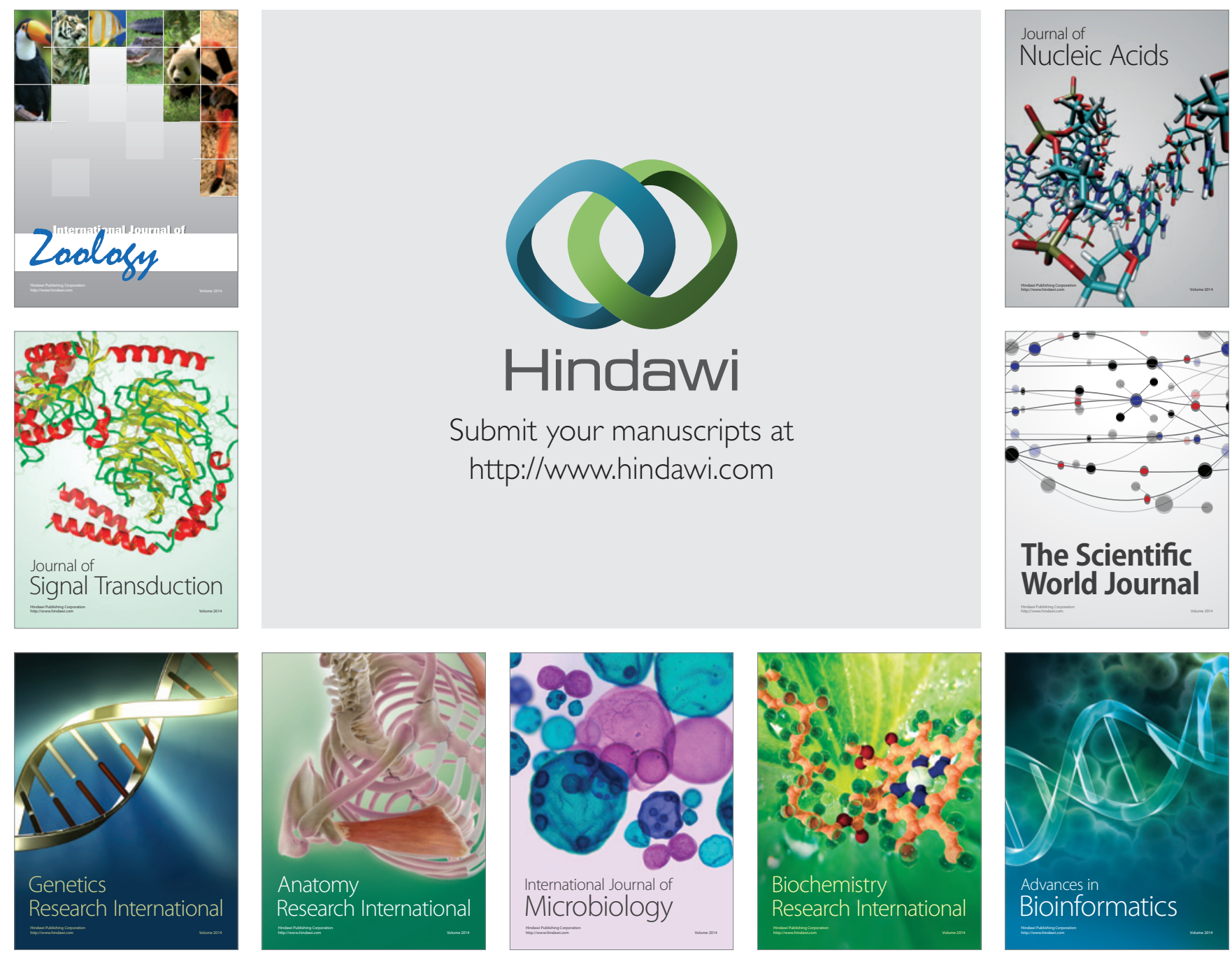

The Scientific World Journal
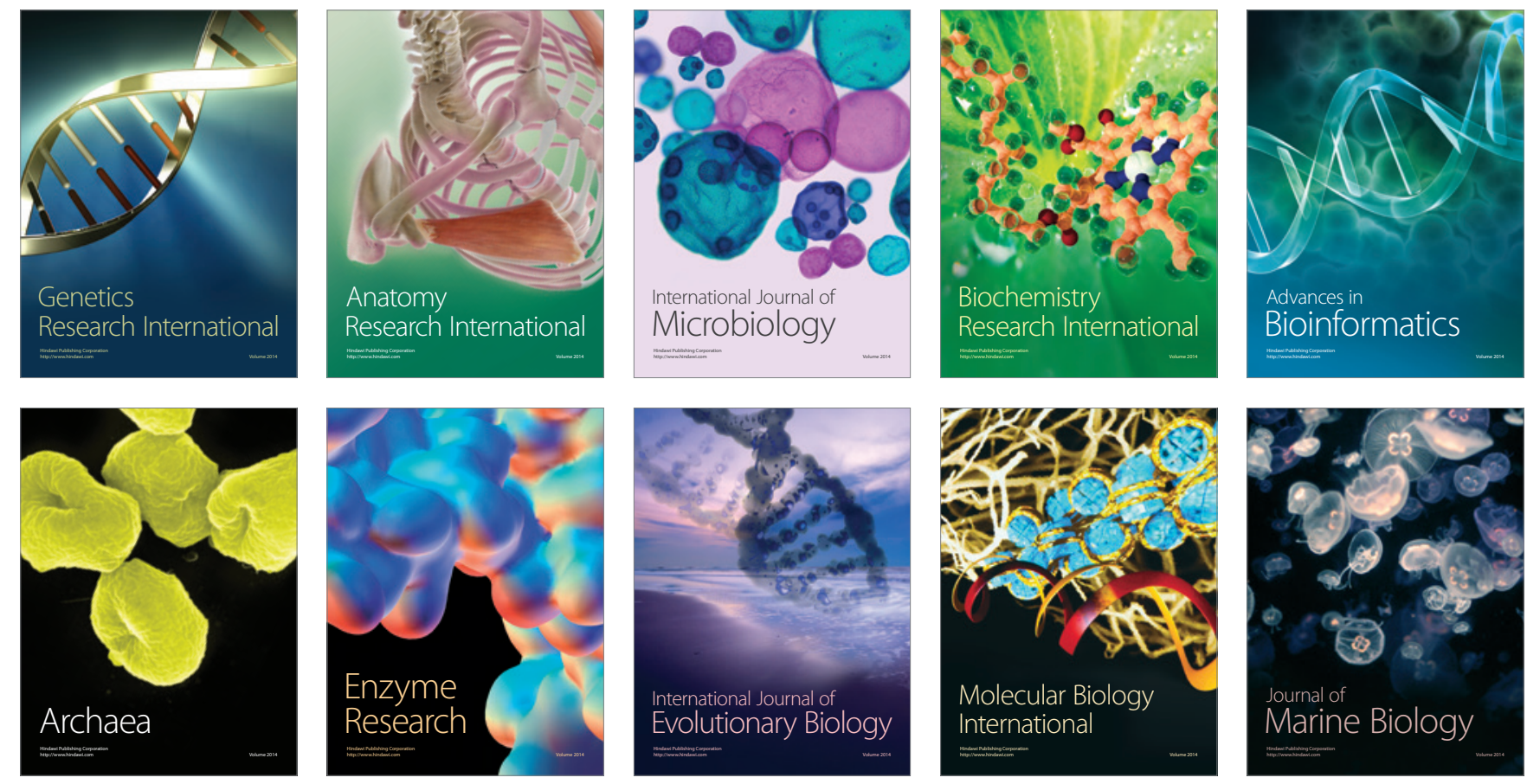